\title{
THE EQUIVALENT MARTINGALE MEASURE CONDITIONS IN A GENERAL MODEL FOR INTEREST RATES
}

\author{
KAIS HAMZA, ${ }^{* * *}$ Monash University \\ SAUL JACKA, ${ }^{* * *}$ University of Warwick \\ FIMA KLEBANER, ${ }^{* * * *}$ Monash University
}

\begin{abstract}
Assuming that the forward rates $f_{t}^{u}$ are semimartingales, we give conditions on their components under which the discounted bond prices are martingales. To achieve this, we give sufficient conditions for the integrated processes $\bar{f}_{t}^{u}=\int_{0}^{u} f_{t}^{v} \mathrm{~d} v$ to be semimartingales, and identify their various components. We recover the no-arbitrage conditions in models well known in the literature and, finally, we formulate a new random field model for interest rates and give its equivalent martingale measure (no-arbitrage) condition.
\end{abstract}

Keywords: No arbitrage; forward rate; semimartingale; Heath-Jarrow-Morton conditions

2000 Mathematics Subject Classification: Primary 60H15

\section{Introduction}

We consider a model for bond markets, based on the forward rates, in which the forward rates are semimartingales. Heath et al. (1990), (1992) considered diffusion models for the forward rates, Shirakawa (1991) introduced jumps into the model, and more general jump diffusion models were introduced by Björk et al. (1997a), (1997b). Kennedy (1994) considered a Gaussian Markov field model for interest rates. In these papers a lot of attention was devoted to finding no-arbitrage conditions for the models introduced. The semimartingale model is the most general one if pricing is done according to the principle of no arbitrage, and includes all of the above models as particular cases. Since the concept of 'no arbitrage', or 'no free lunch', is appealing from the financial point of view, the models considered should not allow for 'free lunches'. The no-arbitrage concept is easy to define in simple models of finitely many assets in discrete time, and the result is known as the first fundamental theorem of asset pricing. This asserts that having no arbitrage is equivalent to the existence of the equivalent martingale measure (the EMM property), the measure under which the discounted security prices are martingales. It is harder to define the concept of no arbitrage in more general models; for example, in Björk et al. (1997a), (1997b), this concept was defined by using measurevalued portfolios and function-valued processes, to reflect the fact that in bond markets there are infinitely many (continuum) traded securities (i.e. bonds parametrized by their maturity). In

Received 11 December 2002; revision received 15 November 2004.

* Postal address: Department of Mathematics, Monash University, Clayton, Victoria 3800, Australia.

** Email address: kais.hamza@sci.monash.edu.au

*** Postal address: Department of Statistics, University of Warwick, Coventry CV4 7AL, UK.

Email address: s.d.jacka@warwick.ac.uk

**** Email address: fima.klebaner@sci.monash.edu.au 
continuous time, the EMM property is an essential assumption of the models; see, e.g. Harrison and Pliska (1981) and Shiryaev (1999). Stricker (1990) and Delbaen and Schachermayer (1994) showed that, in the models with finitely many assets, "no free lunch with bounded risk' is equivalent to the EMM property. They also showed that the EMM property implies no arbitrage. The semimartingale model is the most general one with respect to no-arbitrage pricing since, if the security price process is not a semimartingale, then the market model admits arbitrage opportunities; on the other hand, if the security price process is locally bounded and satisfies the 'no free lunch with vanishing risk' property for simple integrands, then it must be a semimartingale (Delbaen and Schachermayer (1994)).

We will not go any further into definitions of the no-arbitrage concept and their relations to the EMM property (the reader can find a list of results in Shiryaev (1999)), but rather assume the EMM property and find its implications for the coefficients in the model. Often, in practice and in the literature, the EMM conditions are in fact called the 'no-arbitrage conditions', so we will use these terms interchangeably.

After giving the general result Theorem 2.3, we show how the EMM (no-arbitrage) conditions in well-known models in the literature (e.g. the HJM model (Heath et al. (1990), (1992)), the Kennedy Gaussian field model (Kennedy (1994)), and the BDMKR model (Björk et al. (1997b))) are recovered from our condition. Finally, we formulate a new random field model for interest rates and give its EMM conditions.

To obtain the EMM conditions, we must work with integrated semimartingales of forward rates $\bar{f}_{t}^{u}=\int_{0}^{u} f_{t}^{v} \mathrm{~d} v$. To this end, we give conditions for these processes to be semimartingales, and identify their various components. This contribution of the paper is of independent interest (in some of the literature the semimartingale property of the integrated semimartingales is simply assumed).

\section{Semimartingale model for forward rates}

We assume the existence of a continuum of bonds $P(t, T), 0 \leq t \leq T$, where $T$ is a fixed horizon. We also assume the existence of a jointly measurable collection of infinitesimal forward rates $\breve{f}_{t}^{u} \equiv \breve{f}(t, u), 0 \leq t \leq u \leq T$, implied by the bond at $t$ with delivery at $u$.

The forward rates and bonds are related by the identity

$$
P(t, u)=\exp \left\{-\int_{t}^{u} \breve{f}(t, v) \mathrm{d} v\right\} .
$$

The bond market is said to have the EMM property (or to satisfy the EMM condition) if there exists a probability measure $\mathrm{Q} \sim \mathrm{P}$ such that, for each $u \leq T$, the discounted bond price process

$$
S_{t}^{u} \equiv S(t, u)=\exp \left\{-\int_{0}^{t} \breve{f}(s, s) \mathrm{d} s\right\} P(t, u)
$$

is a Q-martingale. As usual, we have assumed the existence of a filtered probability space $\left(\Omega, \mathcal{F}, \mathcal{F}_{t}, \mathrm{P}\right)$ satisfying the usual conditions, i.e. each $\sigma$-algebra is complete and the filtration is right-continuous.

We observe that the EMM condition can be restated as

$$
S(t, u)=\mathrm{E}_{\mathrm{Q}}\left[\exp \left\{-\int_{0}^{u} \breve{f}(v, v) \mathrm{d} v\right\} \mid \mathcal{F}_{t}\right] .
$$

As Musiela and Sondermann (1993) pointed out, the 'HJM' parametrisation of the forward rate curve is problematic in the sense that its domain of definition is not rectangular. Those 
authors dealt with this problem by introducing the time to maturity as an independent variable. We suggest extending the definition of $\breve{f}$ by letting

$$
f(t, u)=\breve{f}(t \wedge u, u) \quad \text { for all } t \text { and all } u \text {. }
$$

Now (2.2) can be rewritten as

$$
S_{t}^{u}=\exp \left\{-\int_{0}^{u} f(t, v) \mathrm{d} v\right\} .
$$

It is intuitively clear that, by applying the logarithmic transformation to $S_{t}^{u}$ and then differentiating, we find that, under the EMM condition, $f_{t}^{u}$, as a process in $t$, must be a semimartingale. Before going any further with this model, we show that, under the EMM condition, the 'discounted forward rate' process is a Q-martingale; as a consequence, we establish that $f_{t}^{u}$ must be a semimartingale. More precisely, let

$$
\phi_{t}^{u} \equiv \phi(t, u)=f_{t}^{u} S_{t}^{u} .
$$

Here, $\phi_{t}^{u}$ represents today's 'value' of the infinitesimal forward rate available at $t$ for a contract straddling $u$.

Theorem 2.1. Assume that $\phi_{t}^{u} \geq 0$ for all $t$ and $u$. Then the market has the EMM property if and only if there exists $a \mathrm{Q} \sim \mathrm{P}$ such that

(i) for Lebesgue almost all $u \in[0, T], \phi_{t}^{u}$, as a process in $t$, is a Q-martingale; and

(ii) for all $t, u \in[0, T], \int_{0}^{u} \phi(t, v) \mathrm{d} v$ is $\mathrm{Q}$-integrable.

Proof. First we observe that $S(t, u)=\exp \left\{-\int_{0}^{u} f(t, v) \mathrm{d} v\right\}$, as a function of $u$, is Lebesgue almost everywhere differentiable, with

$$
\lim _{\varepsilon \rightarrow 0} \frac{S(t, u+\varepsilon)-S(t, u)}{\varepsilon}=-f(t, u) S(t, u)=-\phi(t, u) \quad \text { (Lebesgue almost everywhere), }
$$

which, since $S(t, 0)=1$, translates into

$$
S(t, u)=1-\int_{0}^{u} \phi(t, v) \mathrm{d} v .
$$

Assuming that parts (i) and (ii) hold, and using the conditional Fubini theorem, we obtain

$$
\begin{aligned}
\mathrm{E}_{\mathrm{Q}}\left[S(t, u) \mid \mathcal{F}_{S}\right] & =\mathrm{E}_{\mathrm{Q}}\left[1-\int_{0}^{u} \phi(t, v) \mathrm{d} v \mid \mathcal{F}_{s}\right] \\
& =1-\int_{0}^{u} \mathrm{E}_{\mathrm{Q}}\left[\phi(t, v) \mid \mathcal{F}_{S}\right] \mathrm{d} v \\
& =1-\int_{0}^{u} \phi(s, v) \mathrm{d} v \\
& =S(s, u) .
\end{aligned}
$$


Conversely, if $S$ is a Q-martingale then, by the conditional Fatou lemma,

$$
\begin{aligned}
\phi(s, u) & =-\lim _{\varepsilon \rightarrow 0} \frac{S(s, u+\varepsilon)-S(s, u)}{\varepsilon} \\
& =\lim _{\varepsilon \rightarrow 0} \frac{1}{\varepsilon}\left\{\mathrm{E}_{\mathrm{Q}}\left[S(t, u) \mid \mathcal{F}_{s}\right]-\mathrm{E}_{\mathrm{Q}}\left[S(t, u+\varepsilon) \mid \mathcal{F}_{s}\right]\right\} \\
& \geq \mathrm{E}_{\mathrm{Q}}\left[\lim _{\varepsilon \rightarrow 0} \frac{S(t, u)-S(t, u+\varepsilon)}{\varepsilon} \mid \mathcal{F}_{s}\right]=\mathrm{E}_{\mathrm{Q}}\left[\phi(t, u) \mid \mathcal{F}_{s}\right]
\end{aligned}
$$

and $\phi(t, u)$ is a Q-supermartingale.

On the other hand,

$$
\begin{aligned}
S(0, u) & =\mathrm{E}_{\mathrm{Q}}[S(u, u)] \\
& =\mathrm{E}_{\mathrm{Q}}\left[1-\int_{0}^{u} \phi(u, v) \mathrm{d} v\right] \\
& =1-\int_{0}^{u} \mathrm{E}_{\mathrm{Q}}[\phi(u, v)] \mathrm{d} v \\
& \geq 1-\int_{0}^{u} \phi(0, v) \mathrm{d} v \\
& =S(0, u),
\end{aligned}
$$

so we must have equality in (2.4), from which we conclude that

$$
\mathrm{E}_{\mathrm{Q}}[\phi(u, v)]=\phi(0, v) \quad \text { (Lebesgue almost everywhere). }
$$

Together with the fact that $\phi(t, u)$ is a Q-supermartingale, this implies that $\phi(t, u)$ is a Q-martingale.

Remark 2.1. The positivity requirement in Theorem 2.1 can clearly be relaxed to the assumption that the forward rates are bounded below locally in $t$ and uniformly in $u$.

Corollary 2.1. If the market has the EMM property then

$$
\phi_{t}^{u}=\mathrm{E}_{\mathrm{Q}}\left[f(u, u) \exp \left(-\int_{0}^{u} f(v, v) \mathrm{d} v\right) \mid \mathcal{F}_{t}\right] .
$$

Corollary 2.2. If the market has the EMM property then $S_{t}^{u}$, as a process in $t$, is a semimartingale.

Proof of Corollary 2.2. It follows from Theorem 2.1 that, under the EMM condition, $f_{t}^{u}=\phi_{t}^{u} / S_{t}^{u}$ is a semimartingale (with respect to $\mathrm{Q}$ and, therefore, with respect to $\mathrm{P}$ ).

Next, for each $u \leq T$ we write the canonical representation under $\mathrm{Q}$ of the semimartingale $f_{t}^{u}$, as a process in $t$, as

$$
f^{u}=f_{0}^{u}+M^{u}+B^{u}+h(x) *\left(\mu^{u}-v^{u}\right)+(x-h(x)) * \mu^{u},
$$

where $M^{u}$ is a continuous local martingale; $B^{u}$ is a predictable process of finite variation; $h(x)$ is a bounded function, with compact support, satisfying $h(x)=x$ in a neighbourhood of 0 (e.g. $\left.h(x)=x \mathbf{1}_{\{|x| \leq 1\}}\right) ; \mu^{u}$ is the jump measure of $f^{u}$, i.e.

$$
\mu^{u}(\mathrm{~d} t, \mathrm{~d} x)=\sum_{\left\{s: \Delta f_{s}^{u} \neq 0\right\}} \varepsilon_{\left(s, \Delta f_{s}^{u}\right)}(\mathrm{d} t, \mathrm{~d} x)
$$


(where, as usual, for any càdlàg process $X$, we write $\Delta X_{t}=X_{t}-X_{t-}$ ); $v^{u}$ is the compensator of $\mu^{u}$; and where we have used the notation

$$
(J * \mu)_{t}=(J(x) * \mu)_{t}=\int_{0}^{t} \int_{\mathbb{R}} J(s, x) \mu(\mathrm{d} s, \mathrm{~d} x)
$$

(see Liptser and Shiryaev (1989, p. 189) and Jacod and Shiryaev (1987, p. 84)).

As suggested by (2.1) and (2.2), the semimartingales $f^{u}$ will be integrated with respect to $u$. For this to be possible, we first assume that all of the processes arising in (2.5) are integrable in $u$. We will not list the required assumptions here since, in order to carry out the calculations needed in this paper, more stringent conditions will in fact be imposed on these processes; these conditions will be stated in the results below.

Thus, assuming that they exist, we introduce the following integrated processes, where $A$ is any Borel set in $\mathbb{R}$ :

$$
\begin{aligned}
\bar{f}_{t}^{u} & =\int_{0}^{u} f_{t}^{v} \mathrm{~d} v, \quad \bar{B}_{t}^{u}=\int_{0}^{u} B_{t}^{v} \mathrm{~d} v, \quad \bar{M}_{t}^{u}=\int_{0}^{u} M_{t}^{v} \mathrm{~d} v, \\
\bar{\mu}^{u}(\mathrm{~d} t, \mathrm{~d} x) & =\int_{0}^{u} \mu^{v}(\mathrm{~d} t, \mathrm{~d} x) \mathrm{d} v \\
\bar{v}^{u}(\mathrm{~d} t, \mathrm{~d} x) & =\int_{0}^{u} v^{v}(\mathrm{~d} t, \mathrm{~d} x) \mathrm{d} v .
\end{aligned}
$$

As a general rule, a 'bar' will signify an integration with respect to $u$. Also, let $m^{u}(\mathrm{~d} t, \mathrm{~d} x)$ be the jump measure of $\bar{f}_{t}^{u}$ and $n^{u}(\mathrm{~d} t, \mathrm{~d} x)$ its compensator.

The following theorem gives sufficient conditions under which $\bar{f}^{u}$ is a semimartingale, and identifies its various components.

Theorem 2.2. Assume that

$$
\int_{0}^{T}\left\langle M^{u}, M^{u}\right\rangle_{T} \mathrm{~d} u<\infty ;
$$

(FV) $\quad \int_{0}^{T} A_{T}^{u} \mathrm{~d} u<\infty$, where $A^{u}$ denotes the total-variation process of $B^{u}$;

(J1) $\quad \int_{0}^{T}\left(\int_{0}^{t} \int_{\mathbb{R}} h(x)^{2} v^{u}(\mathrm{~d} s, \mathrm{~d} x)\right) \mathrm{d} u$ is locally integrable; and

$$
\int_{0}^{T}\left(\int_{0}^{T} \int_{\mathbb{R}}|x-h(x)| \mu^{u}(\mathrm{~d} t, \mathrm{~d} x)\right) \mathrm{d} u<\infty .
$$

Then

$$
\bar{f}^{u}=\bar{f}_{0}^{u}+\bar{M}^{u}+\bar{B}^{u}+\int_{0}^{u} h *\left(\mu^{v}-v^{v}\right) \mathrm{d} v+(x-h(x)) * \bar{\mu}^{u},
$$

where $\bar{M}^{u}$ is a continuous, locally square-integrable martingale with sharp bracket

$$
\left\langle\bar{M}^{u}, \bar{M}^{u}\right\rangle_{t}=\int_{0}^{u} \int_{0}^{u}\left\langle M^{v}, M^{w}\right\rangle_{t} \mathrm{~d} v \mathrm{~d} w,
$$

$\bar{B}^{u}$ is predictable and of finite variation, and $\int_{0}^{u} h *\left(\mu^{v}-v^{v}\right) \mathrm{d} v$ is a purely discontinuous local martingale. Furthermore, $\bar{M}^{u}$ is the continuous martingale part of the semimartingale $\bar{f}^{u}$, the jumps of which are given by

$$
\Delta \bar{f}_{t}^{u}=\int_{0}^{u} \Delta f_{t}^{v} \mathrm{~d} v
$$


Proof. Propositions A.2 and A.6 of Appendix A deal with the first part of the theorem. Here, we show the decomposition of $\bar{f}^{u}$. Since $X^{v}:=h *\left(\mu^{v}-v^{v}\right)$ is a purely discontinuous local martingale, its square bracket is

$$
\begin{aligned}
{\left[X^{v}, X^{v}\right]_{t} } & =\sum_{s \leq t}\left[h\left(\Delta f_{s}^{v}\right)-\int_{\mathbb{R}} h(x) v^{v}(\{s\} \times \mathrm{d} x)\right]^{2} \\
& \leq 2 \sum_{s \leq t}\left[h\left(\Delta f_{s}^{v}\right)^{2}+\left(\int_{\mathbb{R}} h(x) v^{v}(\{s\} \times \mathrm{d} x)\right)^{2}\right] \\
& \leq 2 \sum_{s \leq t}\left[h\left(\Delta f_{s}^{v}\right)^{2}+\int_{\mathbb{R}} h(x)^{2} v^{v}(\{s\} \times \mathrm{d} x)\right] \\
& \leq 2\left\{\int_{0}^{t} \int_{\mathbb{R}} h(x)^{2} \mu^{v}(\mathrm{~d} s, \mathrm{~d} x)+\int_{0}^{t} \int_{\mathbb{R}} h(x)^{2} v^{v}(\mathrm{~d} s, \mathrm{~d} x)\right\},
\end{aligned}
$$

where we have used the fact that $v^{v}(\{s\} \times \mathbb{R}) \leq 1$.

Furthermore, the local integrability of $\int_{0}^{u}\left(\int_{0}^{t} \int_{\mathbb{R}} h(x)^{2} \mu^{v}(\mathrm{~d} t, \mathrm{~d} x)\right) \mathrm{d} v$ is equivalent to that of $\int_{0}^{u}\left(\int_{0}^{t} \int_{\mathbb{R}} h(x)^{2} v^{v}(\mathrm{~d} t, \mathrm{~d} x)\right) \mathrm{d} v$ and part (J1) implies that of $\int_{0}^{u}\left[X^{v}, X^{v}\right]_{t} \mathrm{~d} v$, which is a process in $t$. From Proposition A.7, we now conclude that $\int_{0}^{u} X_{t}^{v} \mathrm{~d} v$ is a purely discontinuous local martingale with jumps given by $\int_{0}^{u} \Delta X_{t}^{v} \mathrm{~d} v$.

Finally, since $\Delta B_{t}^{v}=\int_{\mathbb{R}} h(x) v^{v}(\{t\} \times \mathrm{d} x)$, we have

$$
\begin{aligned}
\Delta \bar{f}_{t}^{u}= & \int_{0}^{u} \int_{\mathbb{R}} h(x) v^{v}(\{t\} \times \mathrm{d} x) \mathrm{d} v+\int_{0}^{u}\left(h\left(\Delta f_{t}^{v}\right)-\int_{\mathbb{R}} h(x) v^{v}(\{t\} \times \mathrm{d} x)\right) \mathrm{d} v \\
& +\int_{0}^{u}\left(\Delta f_{t}^{v}-h\left(\Delta f_{t}^{v}\right)\right) \mathrm{d} v \\
= & \int_{0}^{u} \Delta f_{t}^{v} \mathrm{~d} v .
\end{aligned}
$$

Corollary 2.3. In Theorem 2.2, we may replace part (J1) by either

$$
\int_{0}^{T}\left(\int_{0}^{T} \int_{\mathbb{R}} h(x)^{2} v^{u}(\mathrm{~d} t, \mathrm{~d} x)\right) \mathrm{d} u<\infty, \text { or }
$$

$\left(\mathrm{J} 1^{\prime \prime}\right) \quad \int_{0}^{T}\left(\int_{0}^{T} \int_{\mathbb{R}}|h(x)| v^{u}(\mathrm{~d} t, \mathrm{~d} x)\right) \mathrm{d} u$ is locally integrable.

Proof. Define the sequence of stopping times

$$
T_{n}=\inf \left\{t, \int_{0}^{T}\left(\int_{0}^{t} \int_{\mathbb{R}} h(x)^{2} v^{u}(\mathrm{~d} t, \mathrm{~d} x)\right) \mathrm{d} u>n\right\} .
$$

Then, by monotone convergence,

$$
\begin{aligned}
& \int_{0}^{T}\left(\int_{0}^{T_{n}} \int_{\mathbb{R}} h(x)^{2} v^{u}(\mathrm{~d} t, \mathrm{~d} x)\right) \mathrm{d} u \\
& \quad=\lim _{\varepsilon \downarrow 0} \int_{0}^{T}\left(\int_{0}^{T_{n}-\varepsilon} \int_{\mathbb{R}} h(x)^{2} v^{u}(\mathrm{~d} t, \mathrm{~d} x)\right) \mathrm{d} u+\int_{0}^{T} \int_{\mathbb{R}} h(x)^{2} v^{u}\left(\left\{T_{n}\right\} \times \mathrm{d} x\right) \mathrm{d} u \\
& \quad \leq n+T \sup _{x} h(x)^{2}
\end{aligned}
$$

and part (J1) follows. Part (J1") also clearly implies part (J1) since $h(x)^{2} \leq$ const $\times|h(x)|$. 
Theorem 2.3. Assume that (M), (FV), (J1), and (J2) hold, and that

(D) $\quad\left(\mathrm{e}^{-x}+x-1\right) * n^{T}$ is locally integrable.

Then, the discounted bond price process $S_{t}^{u}$ given in (2.2) is a Q-martingale (i.e. the bond market has the EMM property) if and only if

$$
\begin{aligned}
& \frac{1}{2} \int_{0}^{u} \int_{0}^{u}\left\langle M^{v}, M^{w}\right\rangle_{t} \mathrm{~d} v \mathrm{~d} w \\
& \quad=\int_{0}^{u} B_{t}^{v} \mathrm{~d} v+\int_{0}^{u}\left((x-h(x)) * v^{v}\right)_{t} \mathrm{~d} v-\left(\left(\mathrm{e}^{-x}+x-1\right) * n^{u}\right)_{t} .
\end{aligned}
$$

Before we prove the above theorem, we make the following remarks.

Remarks 2.2. 1. Condition (J1") gives a meaning to the otherwise undefined processes $h * \mu^{u}$ and $h * v^{u}$. As a consequence, representation (2.5) simplifies to

$$
f^{u}=f_{0}^{u}+M^{u}+V^{u}+x * \mu^{u},
$$

where $M^{u}$ is a continuous local martingale, $V^{u}$ is a continuous process of finite variation, and $\mu^{u}$ is again the jump measure of $f^{u}$. The two representations are then related by

$$
V_{t}^{u}=B_{t}^{u}-\left(h * v^{u}\right)_{t},
$$

and condition (2.6) becomes

$$
\frac{1}{2} \int_{0}^{u} \int_{0}^{u}\left\langle M^{v}, M^{w}\right\rangle_{t} \mathrm{~d} v \mathrm{~d} w=\int_{0}^{u} V_{t}^{v} \mathrm{~d} v+\int_{0}^{u}\left(x * v^{v}\right)_{t} \mathrm{~d} v-\left(\left(\mathrm{e}^{-x}+x-1\right) * n^{u}\right)_{t} .
$$

Furthermore, $x * \bar{\mu}^{u}=x * \bar{m}^{u}$ and $x * \bar{v}^{u}=x * \bar{n}^{u}$, which lead to the following simplification of (2.8):

$$
\frac{1}{2} \int_{0}^{u} \int_{0}^{u}\left\langle M^{v}, M^{w}\right\rangle_{t} \mathrm{~d} v \mathrm{~d} w=\int_{0}^{u} V_{t}^{v} \mathrm{~d} v-\left(\left(\mathrm{e}^{-x}-1\right) * n^{u}\right)_{t} .
$$

2. If $\bar{f}^{u}$ is continuous ( $\left.n^{u}=0\right)$, then differentiating (2.6) with respect to $u$ gives

$$
\int_{0}^{u}\left\langle M^{u}, M^{v}\right\rangle_{t} \mathrm{~d} v=B_{t}^{u}+\left((x-h(x)) * v^{u}\right)_{t} .
$$

3. If the $f^{u}$ are continuous then $V_{t}^{u}$ is simply $B_{t}^{u}$, and condition (2.6) becomes

$$
B_{t}^{u}=V_{t}^{u}=\int_{0}^{u}\left\langle M^{u}, M^{v}\right\rangle_{t} \mathrm{~d} v
$$

(by differentiating).

4. It is worth commenting on how and why the proposed model is different from those of Heath et al. (1990), (1992) and Björk et al. (1997b): to take full advantage of our model, the filtration used has to be large enough to contain infinitely many Brownian motions. Indeed, if the filtration in use reduces to one generated by only finitely many Brownian motions then, thanks to the predictable representation property, our model would be no different to those of Heath et al. (1990), (1992) and Björk et al. (1997b). For example, our model allows for processes driven by a Brownian sheet rather than by an $n$-dimensional Brownian motion. Likewise, the underlying jump process allowed by our model could be as rich (information-wise) as a Poisson sheet (or infinitely many independent Poisson processes). 
5. As Cont (1998) pointed out, the HJM model can be criticized for being an infinite-dimensional diffusion driven by a finite number of independent noises. The same criticism applies to the BDMKR model in that it is an infinite-dimensional jump diffusion process driven by a finite number of independent noises. Cont suggested modelling the forward curves with an infinitedimensional diffusion driven by an infinite-dimensional Brownian motion. The latter is defined by a family $\mathcal{W}_{t}$ of random linear functionals on a Hilbert space $H$ (say $L^{2}([0, \infty))$ ) such that

(a) $(1 /|\psi|) W_{t}(\psi)$ is a one-dimensional Brownian motion for all $\psi \in H$; and

(b) the Brownian motions $\mathcal{W}_{t}(\psi)$ and $\mathcal{W}_{t}\left(\psi^{\prime}\right)$ are independent for all $\psi, \psi^{\prime} \in H$ with $\psi \perp \psi^{\prime}$.

We emphasize here that the Brownian sheet $W$ introduced in Example 3.4, below, encompasses such an infinite-dimensional noise; to see this, set

$$
\mathcal{W}_{t}(\psi)=\int_{0}^{\infty} \psi(u) W(t, \mathrm{~d} u) .
$$

Proof of Theorem 2.3. The process $\bar{f}_{t}^{u}$ can be written as

$$
\bar{f}^{u}=\bar{f}_{0}^{u}+\bar{M}^{u}+\bar{B}^{u}+h *\left(\bar{\mu}^{u}-\bar{v}^{u}\right)+(x-h(x)) * \bar{\mu}^{u},
$$

where $\bar{M}^{u}$ is a continuous, locally square-integrable martingale, $\bar{B}^{u}$ is predictable and locally integrable, $h *\left(\bar{\mu}^{u}-\bar{v}^{u}\right)$ is a purely discontinuous local martingale, and $\bar{v}^{u}$ compensates $\bar{\mu}^{u}$. Note that $\bar{M}^{u}$ is the continuous martingale part of the semimartingale $\bar{f}^{u}$.

Combining (2.2) with (2.1) and (2.3) leads to

$$
S_{t}^{u}=S_{0}^{u} \exp \left\{-\int_{0}^{u} f(t, v) \mathrm{d} v\right\}=S_{0}^{u} \exp \left\{-\bar{f}_{t}^{u}\right\}
$$

By Itô's formula,

$$
\mathrm{d} S_{t}^{u}=-S_{t-}^{u}\left(\mathrm{~d} \bar{f}_{t}^{u}-\frac{1}{2} \mathrm{~d}\left\langle\bar{M}^{u}, \bar{M}^{u}\right\rangle_{t}-\left[\mathrm{e}^{-\Delta \bar{f}_{t}^{u}}+\Delta \bar{f}_{t}^{u}-1\right]\right) .
$$

Hence, $S^{u}$ is the stochastic exponential of the process

$$
\mathrm{d} X_{t}^{u}=-\mathrm{d} \bar{f}_{t}^{u}+\frac{1}{2} \mathrm{~d}\left\langle\bar{M}^{u}, \bar{M}^{u}\right\rangle_{t}+\mathrm{d}\left(\left(\mathrm{e}^{-x}+x-1\right) * m^{u}\right)_{t}
$$

and, as such, is a local martingale if and only if $X^{u}$ itself is a local martingale. Using (2.11), we see that this happens if and only if the process with differential

$$
\mathrm{d} \bar{B}_{t}^{u}-\frac{1}{2} \mathrm{~d}\left\langle\bar{M}^{u}, \bar{M}^{u}\right\rangle_{t}+\mathrm{d}\left((x-h(x)) * \bar{\mu}^{u}\right)_{t}-\mathrm{d}\left(\left(\mathrm{e}^{-x}+x-1\right) * m^{u}\right)_{t}
$$

is a local martingale.

Making use of assumptions (J2) and (D), we see that, for $S^{u}$ to be a local martingale, it is necessary and sufficient that the process with differential

$$
\mathrm{d} \bar{B}_{t}^{u}-\frac{1}{2} \mathrm{~d}\left\langle\bar{M}^{u}, \bar{M}^{u}\right\rangle_{t}+\mathrm{d}\left((x-h(x)) * \bar{v}^{u}\right)_{t}-\mathrm{d}\left(\left(\mathrm{e}^{-x}+x-1\right) * n^{u}\right)_{t}
$$

be a local martingale. However, this process is predictable and of finite variation; therefore, it must be 0 , and we obtain condition (2.6). 


\section{Applications}

This section deals with existing models (in Examples 3.1 to 3.3) by recovering the relevant results from our general approach. It also presents a new family of models (Example 3.6) that we call 'Gaussian and Poisson random field models'. For clarity of exposition, we shall introduce this model step by step: we first study a basic Gaussian random field model (Example 3.4); next, we look at the basic Poisson random field model (Example 3.5); and then we combine the two for a study of the general model.

Example 3.1. (The HJM model (Heath et al. (1992)).) In the HJM model, it is assumed that the forward rates satisfy a diffusion-type stochastic differential equation in $t$ for any $u$ such that $t \leq u \leq T$, i.e.

$$
\mathrm{d} f(t, u)=\alpha(t, u) \mathrm{d} t+\sigma(t, u) \mathrm{d} W(t)
$$

where $W(t)$ is the standard Brownian motion, for suitable $\alpha$ and $\sigma$. Using convention (2.3), we extend this model to all $t, u \in[0, T]$ by setting $\alpha(t, u)=\sigma(t, u)=0$ for $t>u$.

It is clear that (3.1) is a particular case of (2.5) with the martingale and finite-variation parts

$$
M_{t}^{u}=\int_{0}^{t} \sigma(s, u) \mathrm{d} W(s) \quad \text { and } \quad B_{t}^{u}=\int_{0}^{t} \alpha(s, u) \mathrm{d} s,
$$

respectively, and nil jump part.

Now

$$
\left\langle M^{u}, M^{v}\right\rangle_{t}=\int_{0}^{t} \sigma(s, u) \sigma(s, v) \mathrm{d} s,
$$

and our condition $(\mathrm{M})$ becomes

$$
\int_{0}^{T} \int_{0}^{u} \sigma(t, u)^{2} \mathrm{~d} t \mathrm{~d} u=\int_{0}^{T}\left\langle M^{u}, M^{u}\right\rangle_{T} \mathrm{~d} u<\infty .
$$

While this is more restrictive than condition C1 of Heath et al. (1992, p. 80), which only requires the finiteness (not the integrability) of $\int_{0}^{u} \sigma(t, u)^{2} \mathrm{~d} t$, it makes the unpleasant condition C3 of Heath et al. (1992, p. 82) redundant. Theorem 2.3 now states that a necessary and sufficient condition for the existence of an equivalent martingale measure is, for all $t \leq u$,

$$
\int_{0}^{t} \alpha(s, u) \mathrm{d} s=\int_{0}^{u} \int_{0}^{t} \sigma(s, u) \sigma(s, v) \mathrm{d} s \mathrm{~d} v .
$$

Differentiating in $t$, we recover the no-arbitrage condition for this model derived by Heath et al. (1992):

$$
\alpha(t, u)=\sigma(t, u) \int_{t}^{u} \sigma(t, v) \mathrm{d} v .
$$

Example 3.2. (The Gaussian random field model (Kennedy (1994)).) Consider the Gaussian random field model for forward rates

$$
f(t, u)=f(0, u)+V_{t}^{u}+M_{t}^{u} .
$$

Here $M_{t}^{u}=M([0, t] \times[0, u])$, where $M$ is a Gaussian random measure with covariance function $C(s, t, u, v)$. Also, for each $u$, the $V_{t}^{u}$ are deterministic, continuous, and of finite variation. Note that the $M_{t}^{u}$ are continuous Gaussian martingales such that $\mathrm{E}\left[M_{s}^{u} M_{t}^{v}\right]=\operatorname{cov}\left(M_{s}^{u}, M_{t}^{v}\right)=$ $C(s, t, u, v)$, which is a function of $(s \wedge t, u \wedge v)$. In this case, the $f(t, u)$ form a family of continuous semimartingales. 
Now,

$$
\left\langle M^{u}, M^{v}\right\rangle_{t}=\mathrm{E}\left[M_{t}^{u} M_{t}^{v}\right]=C(t, t, u, v) .
$$

Recalling that $f_{t}^{u}=f_{t \wedge u}^{u}$, we recover Kennedy's (1994) no-arbitrage condition, i.e.

$$
V_{t}^{u}=\int_{0}^{u} C(t \wedge v, t \wedge v, u, v) \mathrm{d} v,
$$

from our condition (2.10).

Example 3.3. (The jump diffusion model (Björk et al. (1997a), (1997b)).) In this model it is assumed that the forward rates satisfy a jump diffusion stochastic differential equation in $t$ for any $u \leq T$ with $t \leq u$ (see Björk et al. (1997b, Assumption 5.1)):

$$
\mathrm{d} f(t, u)=\alpha(t, u) \mathrm{d} t+\sigma(t, u) \mathrm{d} W(t)+\int_{\mathbb{R}} \delta(t, x, u)(\lambda(\mathrm{d} t, \mathrm{~d} x)-l(\mathrm{~d} t, \mathrm{~d} x)) .
$$

Here, $W(t)$ is the standard Brownian motion; $\lambda(\mathrm{d} t, \mathrm{~d} x)$ is a jump measure of a semimartingale; $l(\mathrm{~d} t, \mathrm{~d} x)$ is its continuous compensator; and the coefficients $\alpha(t, u), \sigma(t, u)$, and $\delta(t, x, u)$ are continuous in $u$ and predictable in $t$, satisfying the following conditions for all $u, t \leq u \leq T$ :

$$
\begin{array}{r}
\int_{0}^{u}\left(\int_{t}^{u}|\alpha(s, v)| \mathrm{d} v\right) \mathrm{d} s<\infty, \\
\int_{0}^{u}\left(\int_{t}^{u}|\sigma(s, v)|^{2} \mathrm{~d} v\right) \mathrm{d} s<\infty, \\
\int_{0}^{u} \int_{\mathbb{R}}\left(\int_{t}^{u}|\delta(s, x, v)|^{2} \mathrm{~d} v\right) l(\mathrm{~d} s, \mathrm{~d} x)<\infty .
\end{array}
$$

Although this model may seem similar to (2.5), there are fundamental differences that we now underline. First, note that $\delta(t, x, u) \lambda(\mathrm{d} t, \mathrm{~d} x)$ is not the jump measure of $f_{t}^{u}$; it may not even be integer-valued and, therefore, not be a jump measure at all. Model (3.2) generalizes the HJM model by adding a jump component, but in both cases the processes $f_{t}^{u}$ are driven by the same Brownian motion $W(t)$ and the dependence on $u$ is modelled by taking the diffusion coefficient $\sigma(t, u)$ to be a function of $u$. Similarly, in Björk et al. (1997a), (1997b), the jumps of all processes $f_{t}^{u}$ were modelled by the same underlying jump process with jump measure $\lambda(\mathrm{d} t, \mathrm{~d} x)$, and the dependence on $u$ was modelled by taking the jump coefficient $\delta(t, x, u)$ to be a function of $u$. Our model, on the other hand, allows the driving processes (the continuous part as well as the jump part) to depend on $u$. Using convention (2.3), we can extend this model to all $t, u \in[0, T]$ by setting $\alpha(t, u)=\sigma(t, u)=\delta(t, x, u)=0$ for $t>u$.

Let us write the various parameters appearing in our model in terms of those appearing in the jump diffusion model. Let $\xi_{t}$ be the underlying jump process with jump measure $\lambda(\mathrm{d} t, \mathrm{~d} x)$. Then $\Delta f_{t}^{u}=\mathbf{1}_{\left\{\Delta \xi_{t} \neq 0\right\}} \delta\left(t, \Delta \xi_{t}, u\right)$ and

$$
\begin{aligned}
\mu^{u}([0, t] \times A) & =\sum_{s \leq t} \mathbf{1}_{A}\left(\Delta f_{s}^{u}\right) \mathbf{1}_{\left\{\Delta f_{s}^{u} \neq 0\right\}} \\
& =\sum_{s \leq t} \mathbf{1}_{A}\left(\delta\left(s, \Delta \xi_{s}, u\right)\right) \mathbf{1}_{\left\{\Delta \xi_{s} \neq 0\right\}} \\
& =\int_{0}^{t} \int_{\mathbb{R}} \mathbf{1}_{A}(\delta(s, x, u)) \lambda(\mathrm{d} s, \mathrm{~d} x) .
\end{aligned}
$$


We also have $\sum_{s \leq t}\left|\Delta f_{s}^{u}\right|=\int_{0}^{t} \int_{\mathbb{R}}|\delta(s, x, u)| \lambda(\mathrm{d} s, \mathrm{~d} x)$. Therefore, the integrability of $\delta(s, x, u)$ with respect to $\lambda(\mathrm{d} s, \mathrm{~d} x)$, assumed by (3.2), implies that the jumps of $f^{u}$ are summable and, hence, that the simplified model of (2.7) applies. Now, the semimartingale $f_{t}^{u}-\sum_{s \leq t} \Delta f_{s}^{u}$ is continuous and, therefore, special. Identifying the continuous martingale parts and the continuous finite-variation parts, we find that

$$
M_{t}^{u}=\int_{0}^{t} \sigma(s, u) \mathrm{d} W(s) \quad \text { and } \quad V_{t}^{u}=\int_{0}^{t} \alpha(s, u) \mathrm{d} s-\int_{0}^{t} \int_{\mathbb{R}} \delta(s, x, u) l(\mathrm{~d} s, \mathrm{~d} x) .
$$

The final step in our identification procedure is the specification of the compensator $v^{u}$. For any predictable $J(t, x) \geq 0$,

$$
\begin{aligned}
\mathrm{E}\left[\int_{0}^{t} \int_{\mathbb{R}} J(s, x) v^{u}(\mathrm{~d} s, \mathrm{~d} x)\right] & =\mathrm{E}\left[\int_{0}^{t} \int_{\mathbb{R}} J(s, x) \mu^{u}(\mathrm{~d} s, \mathrm{~d} x)\right] \\
& =\mathrm{E}\left[\int_{0}^{t} \int_{\mathbb{R}} J(s, \delta(s, x, u)) \lambda(\mathrm{d} s, \mathrm{~d} x)\right] \\
& =\mathrm{E}\left[\int_{0}^{t} \int_{\mathbb{R}} J(s, \delta(s, x, u)) l(\mathrm{~d} s, \mathrm{~d} x)\right],
\end{aligned}
$$

implying that

$$
v^{u}([0, t] \times A)=\int_{0}^{t} \int_{\mathbb{R}} \mathbf{1}_{A}(\delta(s, x, u)) l(\mathrm{~d} s, \mathrm{~d} x) .
$$

We will also need the jump measure of the integrated process. Taking into account the integrability conditions given in (3.3), the fact that the processes $f^{u}$ are driven by the same Brownian motion (i.e. $W(t)$ ) for the martingale part, the same jump process (i.e. $\xi_{t}$ ) for the jump part, and the same processes of finite variation (i.e. $t$ and $l(\mathrm{~d} t, \mathrm{~d} x))$ lets us write

$$
\mathrm{d} \bar{f}(t, u)=\bar{\alpha}(t, u) \mathrm{d} t+\bar{\sigma}(t, u) \mathrm{d} W(t)+\int_{\mathbb{R}} \bar{\delta}(t, x, u)(\lambda(\mathrm{d} t, \mathrm{~d} x)-l(\mathrm{~d} t, \mathrm{~d} x)),
$$

recalling that a bar signifies integration of the barred quantity with respect to $u$.

This automatically provides us with the jumps of $\bar{f}^{u}$,

$$
\Delta \bar{f}_{t}^{u}=\mathbf{1}_{\left\{\Delta \xi_{t} \neq 0\right\}} \bar{\delta}\left(t, \Delta \xi_{t}, u\right),
$$

and, ultimately, with the jump measure

$$
m^{u}([0, t] \times A)=\int_{0}^{t} \int_{\mathbb{R}} \mathbf{1}_{A}(\bar{\delta}(s, x, u)) \lambda(\mathrm{d} s, \mathrm{~d} x)
$$

and its compensator

$$
n^{u}([0, t] \times A)=\int_{0}^{t} \int_{\mathbb{R}} \mathbf{1}_{A}(\bar{\delta}(s, x, u)) l(\mathrm{~d} s, \mathrm{~d} x) .
$$

Before we are able to apply Theorem 2.3, we must check that conditions (M), (FV), (J1), and (J2) are satisfied. Conditions (FV) and (M) are simply the first and second parts of (3.3), respectively. Using (3.4), we can rewrite the third part of (3.3) as $\int_{t}^{u}\left(\int_{0}^{u} \int_{\mathbb{R}} x^{2} v^{v}(\mathrm{~d} s, \mathrm{~d} x)\right) \mathrm{d} v<\infty$, from which condition $\left(\mathrm{J}^{\prime}\right)$ immediately follows. 
Thus, condition (2.6) becomes

$$
\frac{1}{2} \int_{0}^{t} \bar{\sigma}^{2}(s, u) \mathrm{d} s=\int_{0}^{t} \bar{\alpha}(s, u) \mathrm{d} s-\int_{0}^{t} \int_{\mathbb{R}}\left(\mathrm{e}^{-\bar{\delta}(s, x, u)}+\bar{\delta}(s, x, u)-1\right) l(\mathrm{~d} s, \mathrm{~d} x),
$$

which is the condition obtained in Björk et al. (1997b, Equation (5.15)). (Note that their D corresponds to our $-\bar{\delta}$, and their $a$ to our $\frac{1}{2} \bar{\sigma}^{2}-\bar{\alpha}$.) Furthermore, in the particular case where $l(\mathrm{~d} t, \mathrm{~d} x)=k_{t}(\mathrm{~d} x) \mathrm{d} t$, differentiation yields the condition

$$
\frac{1}{2} \bar{\sigma}^{2}(t, u)=\bar{\alpha}(t, u)-\int_{\mathbb{R}}\left(\mathrm{e}^{-\bar{\delta}(t, x, u)}+\bar{\delta}(t, x, u)-1\right) k_{t}(\mathrm{~d} x),
$$

which is condition (5.16) of Björk et al. (1997b). Further, differentiating with respect to $u$ we obtain the condition

$$
\alpha(t, u)=\sigma(t, u) \bar{\sigma}(t, u)+\int_{\mathbb{R}} \delta(t, x, u)\left(1-\mathrm{e}^{-\bar{\delta}(t, x, u)}\right) k_{t}(\mathrm{~d} x)
$$

(see also Björk et al. (1997a, Equation (24))).

Example 3.4. (The basic Gaussian random field model.) Assume that

$$
f_{t}^{u}=f_{0}^{u}+V_{t}^{u}+\int_{0}^{t} \int_{0}^{u} \sigma(s, v) W(\mathrm{~d} s, \mathrm{~d} v)
$$

where $\sigma(t, u)$ is deterministic, $W(t, u)$ is a Brownian sheet, and, for any fixed $u, V_{t}^{u}$ is of finite variation and continuous.

$W$ is a Gaussian random measure on $\mathbb{R}_{+}^{2}$ and, thus, for any pair $(A, B)$ of disjoint Borel sets in $\mathbb{R}_{+}^{2}, W(A)$ and $W(B)$ are independent zero-mean Gaussian random variables with variances $\operatorname{Leb}(A)$ and $\operatorname{Leb}(B)$.

We will make the following assumption about $\sigma(t, u)$ :

$$
\int_{0}^{T} \int_{0}^{T} \int_{0}^{u} \sigma(s, v)^{2} \mathrm{~d} v \mathrm{~d} s \mathrm{~d} u<\infty .
$$

As we will see later, this is simply condition (M). We will also assume that condition (FV) is satisfied. That is, if $A^{u}$ is the total-variation process of $V^{u}$, then

$$
\int_{0}^{T} A_{T}^{u} \mathrm{~d} u<\infty
$$

Since $\sigma$ is deterministic, $M_{t}^{v}=\int_{0}^{t} \int_{0}^{v} \sigma(s, w) W(\mathrm{~d} s, \mathrm{~d} w)$ is a Gaussian martingale with variance $\int_{0}^{t} \int_{0}^{v} \sigma(s, w)^{2} \mathrm{~d} s \mathrm{~d} w$. Furthermore, for $v<v^{\prime}, M_{t}^{v^{\prime}}$ can be written as the sum of the two independent random variables $M_{t}^{v}$ and $\int_{0}^{t} \int_{v}^{v^{\prime}} \sigma(s, w) W(\mathrm{~d} s, \mathrm{~d} w)$. It follows that

$$
\left\langle M^{v}, M^{v^{\prime}}\right\rangle_{t}=\left\langle M^{v \wedge v^{\prime}}, M^{v \wedge v^{\prime}}\right\rangle_{t}=\int_{0}^{t} \int_{0}^{v \wedge v^{\prime}} \sigma(s, w)^{2} \mathrm{~d} w \mathrm{~d} s,
$$

and the EMM condition for this model is given by

$$
\frac{1}{2} \int_{0}^{u} \int_{0}^{u} \int_{0}^{t} \int_{0}^{v \wedge v^{\prime}} \sigma(s, w)^{2} \mathrm{~d} w \mathrm{~d} s \mathrm{~d} v \mathrm{~d} v^{\prime}=\int_{0}^{u} V_{t}^{v} \mathrm{~d} v
$$


Differentiating with respect to $u$, we obtain the following EMM condition:

$$
\int_{0}^{u} \int_{0}^{t} \int_{0}^{v} \sigma(s, w)^{2} \mathrm{~d} w \mathrm{~d} s \mathrm{~d} v=V_{t}^{u}
$$

Note that Kennedy's condition (see Example 3.2) can be recovered from the above by noting that

$$
C(s, t, u, v)=\int_{0}^{s \wedge t} \int_{0}^{u \wedge v} \sigma\left(s^{\prime}, v^{\prime}\right)^{2} \mathrm{~d} s^{\prime} \mathrm{d} v^{\prime}
$$

The no-arbitrage condition in the stochastic partial differential equation model (Hamza and Klebaner (1995)) can also be easily recovered from (3.5).

Example 3.5. (The basic Poisson random field model.) Assume that

$$
f_{t}^{u}=f_{0}^{u}+V_{t}^{u}+\int_{0}^{t} \int_{0}^{u} \lambda(s, v) N(\mathrm{~d} s, \mathrm{~d} v)
$$

where $N(t, u)$ is a Poisson sheet, $\lambda(s, v)$ is deterministic, and, for any fixed $u, V_{t}^{u}$ is of finite variation, is continuous, and satisfies condition (FV).

As for the Brownian sheet, $N$ is a Poisson random measure on $\mathbb{R}_{+}^{2}$ such that, for any pair $(A, B)$ of disjoint Borel sets in $\mathbb{R}_{+}^{2}, N(A)$ and $N(B)$ are independent Poisson random variables with means $\operatorname{Leb}(A)$ and $\operatorname{Leb}(B)$.

We will make the following assumption about $\lambda(s, v)$ :

$$
\int_{0}^{T} \int_{0}^{T} \int_{0}^{u}|\lambda(s, v)| \mathrm{d} v \mathrm{~d} s \mathrm{~d} u<\infty
$$

As we will see later, this is simply the combination of conditions $\left(\mathrm{J} 1^{\prime \prime}\right)$ and $(\mathrm{J} 2)$.

In the sequel, $u$ is fixed. Let $\left(T_{1}, U_{1}\right),\left(T_{2}, U_{2}\right), \ldots$ be the random points defining the restriction of the Poisson sheet $N$ to $[0, u] \times[0, u]$. The numbering of this sequence is such that $T_{n}<T_{n+1}$. For $v \leq u$, let

$N_{t}^{v}=N([0, t] \times[0, v]), \quad Z_{t}^{v}=\int_{0}^{t} \int_{0}^{v} \lambda(s, w) N(\mathrm{~d} s, \mathrm{~d} w)=\sum_{n} \lambda\left(T_{n}, U_{n}\right) \mathbf{1}_{\left\{T_{n} \leq t\right\}} \mathbf{1}_{\left\{U_{n} \leq u\right\}}$.

Then, with

$$
U(t)=U_{n} \quad \text { for } T_{n} \leq t<T_{n+1},
$$

we can write

$$
\Delta f_{t}^{v}=\Delta Z_{t}^{v}=\lambda(t, U(t)) \Delta N_{t}^{v}=\lambda(t, U(t)) \mathbf{1}_{\{U(t) \leq v\}} \Delta N_{t}^{u}
$$

Note that if $\Delta N_{t}^{v}=1$ then $\Delta N_{t}^{u}=1$ for each $u \geq v$. Since $Z_{t}^{v}$, as a process in $t$, has independent increments, the compensator of its jump measure is deterministic. This statement follows from a monotone-class argument identical to that of Jacod and Shiryaev (1987, p. 71). In the computation below, we use the fact that, as a process in $v, N_{s}^{v}$ is Poisson and, therefore, 
that the conditional law of $U(s)$, given the entire path of $N_{\theta}^{u}$ (as a process in $\theta$ ), is uniform on the interval $[0, u]$. Hence,

$$
\begin{aligned}
v^{v}([0, t] \times A) & =\mathrm{E}\left[\mu^{v}([0, t] \times A)\right]=\sum_{s \leq t} \mathrm{P}\left[\Delta Z_{s}^{v} \in A^{*}\right] \quad\left(\text { where } A^{*}=A \backslash\{0\}\right) \\
& =\sum_{s \leq t} \mathrm{P}\left[\lambda(s, U(s)) \mathbf{1}_{\{U(s) \leq v\}} \in A^{*}, \Delta N_{s}^{u} \neq 0\right] \\
& =\sum_{s \leq t} \mathrm{E}\left[\mathrm{P}\left[\lambda(s, U(s)) \mathbf{1}_{\{U(s) \leq v\}} \in A^{*} \mid N_{\theta}^{u}, \theta \geq 0\right] \mathbf{1}_{\left\{\Delta N_{s}^{u} \neq 0\right\}}\right] \\
& =\sum_{s \leq t} \mathrm{E}\left[\frac{1}{u} \int_{0}^{u} \mathbf{1}_{\{w \leq v\}} \mathbf{1}_{A^{*}}(\lambda(s, w)) \mathrm{d} w \mathbf{1}_{\left\{\Delta N_{s}^{u} \neq 0\right\}}\right] \\
& =\frac{1}{u} \int_{0}^{v} \mathrm{E}\left[\sum_{s \leq t} \mathbf{1}_{A^{*}}(\lambda(s, w)) \Delta N_{s}^{u}\right] \mathrm{d} w \\
& =\frac{1}{u} \int_{0}^{v} \mathrm{E}\left[\int_{0}^{t} \mathbf{1}_{A^{*}}(\lambda(s, w)) \mathrm{d} N_{s}^{u}\right] \mathrm{d} w \\
& =\frac{1}{u} \int_{0}^{v} \int_{0}^{t} \mathbf{1}_{A^{*}}(\lambda(s, w)) u \mathrm{~d} s \mathrm{~d} w \\
& =\int_{0}^{t} \int_{0}^{v} \mathbf{1}_{A^{*}}(\lambda(s, w)) \mathrm{d} w \mathrm{~d} s .
\end{aligned}
$$

It follows that, for any $\phi$,

$$
\left(\phi * v^{v}\right)_{t}=\int_{0}^{t} \int_{0}^{v} \phi^{*}(s, \lambda(s, w)) \mathrm{d} w \mathrm{~d} s,
$$

where $\phi^{*}(s, x)=\phi(s, x) \mathbf{1}_{\{x \neq 0\}}$. Note that $\phi^{*}(s, x)=\phi(s, x)$ if $\phi(s, 0)=0$.

We now turn to the jump measure of the integrated process and notice that, $\bar{V}^{u}$ being continuous, the jumps of $\bar{f}^{u}$ are those of $\bar{Z}^{u}$, which we now evaluate:

$$
\bar{Z}_{t}^{u}=\int_{0}^{u}\left(\int_{0}^{t} \int_{0}^{v} \lambda(s, w) N(\mathrm{~d} s, \mathrm{~d} w)\right) \mathrm{d} v=\int_{0}^{t} \int_{0}^{u}(u-w) \lambda(s, w) N(\mathrm{~d} s, \mathrm{~d} w) .
$$

Its jump measure can thus be obtained by simply replacing $\lambda(s, w)$ by $(u-w) \lambda(s, w)$ in (3.6). We find that, for any deterministic $\phi$,

$$
\left(\phi * n^{u}\right)_{t}=\int_{0}^{t} \int_{0}^{u} \phi^{*}(s,(u-w) \lambda(s, w)) \mathrm{d} w \mathrm{~d} s .
$$

Applying (2.8), we then find the following EMM condition:

$$
\begin{aligned}
\int_{0}^{u} V_{t}^{v} \mathrm{~d} v+\int_{0}^{u}\left(x * v^{v}\right)_{t} \mathrm{~d} v & =\left(\left(\mathrm{e}^{-x}+x-1\right) * n^{u}\right)_{t} \\
& =\int_{0}^{t} \int_{0}^{u}\left(\mathrm{e}^{-(u-w) \lambda(s, w)}+(u-w) \lambda(s, w)-1\right) \mathrm{d} w \mathrm{~d} s \\
& =\int_{0}^{t} \int_{0}^{u} \mathrm{e}^{-(u-w) \lambda(s, w)} \mathrm{d} w \mathrm{~d} s+\int_{0}^{u}\left(x * v^{v}\right)_{t} \mathrm{~d} v-t u
\end{aligned}
$$


that is,

$$
\int_{0}^{u} V_{t}^{v} \mathrm{~d} v=\int_{0}^{t} \int_{0}^{u} \mathrm{e}^{-(u-w) \lambda(s, w)} \mathrm{d} w \mathrm{~d} s-t u .
$$

Differentiating this with respect to $u$ gives

$$
V_{t}^{v}=-\int_{0}^{t} \int_{0}^{u} \lambda(s, v) \mathrm{e}^{-(u-v) \lambda(s, v)} \mathrm{d} v \mathrm{~d} s .
$$

Example 3.6. (The Gaussian and Poisson random field model.) Assume that

$$
f_{t}^{u}=f_{0}^{u}+V_{t}^{u}+\int_{0}^{t} H_{s}^{u} \int_{0}^{u} \sigma(s, v) W(\mathrm{~d} s, \mathrm{~d} v)+\int_{0}^{t} K_{s} \int_{0}^{u} \lambda(s, v) N(\mathrm{~d} s, \mathrm{~d} v),
$$

where $\sigma(s, v)$ and $\lambda(s, v)$ are deterministic; $W(t, u)$ is a Brownian sheet; $N(t, u)$ is a Poisson sheet; and, for any fixed $u, V_{t}^{u}$ is of finite variation and continuous, and $H_{t}^{u}$ and $K_{t}$ are predictable.

Theorem 3.1. The EMM condition for this model is given by

$$
\int_{0}^{u} \int_{0}^{t} H_{s}^{u} H_{s}^{v} \int_{0}^{v} \sigma(s, w)^{2} \mathrm{~d} w \mathrm{~d} s \mathrm{~d} v=V_{t}^{u}+\int_{0}^{t} K_{s} \int_{0}^{u} \lambda(s, v) \mathrm{e}^{-(u-v) K_{s} \lambda(s, v)} \mathrm{d} v \mathrm{~d} s .
$$

When there is no continuous martingale part (i.e. $\sigma=0$ or $H=0$ ), (3.7) simplifies to

$$
V_{t}^{u}=-\int_{0}^{t} K_{s} \int_{0}^{u} \lambda(s, v) \mathrm{e}^{-(u-v) K_{s} \lambda(s, v)} \mathrm{d} v \mathrm{~d} s
$$

while, in the absence of jumps $(\lambda=0)$, it simplifies to

$$
\int_{0}^{u} \int_{0}^{t} H_{s}^{u} H_{s}^{v} \int_{0}^{v} \sigma(s, w)^{2} \mathrm{~d} w \mathrm{~d} s \mathrm{~d} v=V_{t}^{u} .
$$

Proof. Let $L_{t}^{u}=\int_{0}^{t} \int_{0}^{u} \sigma(s, v) W(\mathrm{~d} s, \mathrm{~d} v)$, so that

$$
M_{t}^{u}=\int_{0}^{t} H_{s}^{u} \mathrm{~d} L_{s}^{u}
$$

The properties of the Gaussian martingale $L_{t}^{u}$ were discussed in Example 3.4. They imply that

$$
\left\langle M^{u}, M^{u^{\prime}}\right\rangle_{t}=\int_{0}^{t} H_{s}^{u} H_{s}^{u^{\prime}} \int_{0}^{u \wedge u^{\prime}} \sigma(s, v)^{2} \mathrm{~d} v \mathrm{~d} s .
$$

The jump part of $f_{t}^{v}$ can be expressed in terms of the process

$$
Z_{t}^{v}=\int_{0}^{t} \int_{0}^{v} \lambda(s, w) N(\mathrm{~d} s, \mathrm{~d} w),
$$

introduced in Example 3.5, as

$$
Y_{t}^{v}=\int_{0}^{t} K_{s} \mathrm{~d} Z_{s}^{v}
$$


It immediately follows that, with the notation of Example 3.5, the jumps of $f_{t}^{v}$ are given by

$$
\Delta f_{t}^{v}=\Delta Y_{t}^{v}=K_{t} \Delta Z_{t}^{v}=K_{t} \lambda(t, U(t)) \mathbf{1}_{\{U(t) \leq v\}} \Delta N_{t}^{u} .
$$

The compensators $v^{u}$ and $n^{u}$ are then found to be

$$
\begin{aligned}
& v^{u}([0, t] \times A)=\int_{0}^{t} \int_{0}^{u} \mathbf{1}_{A^{*}}\left(K_{s} \lambda(s, v)\right) \mathrm{d} v \mathrm{~d} s, \\
& n^{u}([0, t] \times A)=\int_{0}^{t} \int_{0}^{u} \mathbf{1}_{A^{*}}\left((u-v) K_{s} \lambda(s, v)\right) \mathrm{d} v \mathrm{~d} s .
\end{aligned}
$$

Now the proof is completed by differentiating (2.9) with respect to $u$.

\section{Appendix A.}

In the sequel, we let $\left(\Omega,\left(\mathcal{F}_{t}\right)_{t \geq 0}, \mathrm{P}\right)$ be a stochastic basis on which $M_{t}^{v}$ and $B_{t}^{v}$ are families, indexed by $v \leq u$, of local martingales and processes of finite variation, respectively. Let $A_{t}^{v}$ be the total-variation process of $B_{t}^{v}$, and suppose that $B_{0}^{v}=0$ for almost every $v \leq u$. We also assume that we are given a family of random measures $\mu^{v}$ with compensators $v^{v}$. Results on a finite horizon $[0, T]$ immediately follow by stopping at $T$.

Proposition A.1. Suppose that $B_{t}^{v}$ is a family of increasing processes for which $\int_{0}^{u} B_{t}^{v} \mathrm{~d} v<\infty$. Then $\bar{B}_{t}^{u}=\int_{0}^{u} B_{t}^{v} \mathrm{~d} v$ is an increasing process and $\Delta \bar{B}_{t}^{u}=\int_{0}^{u} \Delta B_{t}^{v} \mathrm{~d} v$.

Proof. By monotone convergence, we see that $\bar{B}_{t-}^{u}=\int_{0}^{u} B_{t-}^{v} \mathrm{~d} v$. The result immediately follows.

Proposition A.2. Suppose that $\int_{0}^{u} A_{t}^{v} \mathrm{~d} v<\infty$. Then $\bar{B}_{t}^{u}=\int_{0}^{u} B_{t}^{v} \mathrm{~d} v$ is a process of finite variation with $\Delta \bar{B}_{t}^{u}=\int_{0}^{u} \Delta B_{t}^{v} \mathrm{~d} v$. If, for almost every $v \leq u, B^{v}$ is predictable, then so is $\bar{B}^{u}$.

Furthermore, if $\int_{0}^{u} A_{t}^{v} \mathrm{~d} v$ is locally integrable and $C_{t}^{v}$ denotes the compensator (or dual predictable projection) of $B_{t}^{v}$, then the compensator of $\bar{B}_{t}^{u}$ is $\bar{C}_{t}^{u}=\int_{0}^{u} C_{t}^{v} \mathrm{~d} v$.

Proof. $B_{t}^{v}=\left(A_{t}^{v}+B_{t}^{v}\right)-A_{t}^{v}$ is a measurable decomposition of $B_{t}^{v}$ as a difference of two increasing processes. Since $\left|B_{t}^{v}\right| \leq A_{t}^{v}$, the integrability condition on $V_{t}^{v}$ implies that $\bar{B}_{t}^{u}=$ $\int_{0}^{u} B_{t}^{v} \mathrm{~d} v=\int_{0}^{u}\left(A_{t}^{v}+B_{t}^{v}\right) \mathrm{d} v-\int_{0}^{u} A_{t}^{v} \mathrm{~d} v$ is a process of finite variation written as the difference of two increasing processes. The jumps of $\bar{B}_{t}^{u}$ are obtained by applying Proposition A.1 to $A_{t}^{v}+B_{t}^{v}$ and $A_{t}^{v}$.

Since the $C_{t}^{v}$ are predictable, the same is true of the approximating sums of $\bar{C}_{t}^{u}$. Consequently $\bar{C}_{t}^{u}$, as a limit of predictable processes, is predictable itself. Since the total variation of $C^{v}$ is smaller than the compensator of $A^{v}$, the local integrability of $\int_{0}^{u} A_{t}^{v} \mathrm{~d} v$, and the above, imply that $\bar{C}_{t}^{u}$ is of finite variation. The rest follows by Fubini's theorem.

Proposition A.3. The compensator of $\bar{\mu}^{u}(\mathrm{~d} t, \mathrm{~d} x)$ is $\bar{\nu}^{u}(\mathrm{~d} t, \mathrm{~d} x)=\int_{0}^{u} v^{v}(\mathrm{~d} t, \mathrm{~d} x) \mathrm{d} v$.

Proof. Since a pointwise limit of predictable processes is itself predictable,

$$
\bar{v}^{u}(\mathrm{~d} t, \mathrm{~d} x)=\int_{0}^{u} v^{v}(\mathrm{~d} t, \mathrm{~d} x) \mathrm{d} v
$$


is predictable. Let $H(t, x) \geq 0$ also be predictable. Then, by Fubini's theorem and the definition of compensators,

$$
\begin{aligned}
\mathrm{E}\left(\int_{0}^{\infty} \int_{\mathbb{R}} H(t, x) \bar{\mu}^{u}(\mathrm{~d} t, \mathrm{~d} x)\right) & =\mathrm{E}\left(\int_{0}^{u}\left(\int_{0}^{\infty} \int_{\mathbb{R}} H(t, x) \mu^{v}(\mathrm{~d} t, \mathrm{~d} x)\right) \mathrm{d} v\right) \\
& =\int_{0}^{u} \mathrm{E}\left(\int_{0}^{\infty} \int_{\mathbb{R}} H(t, x) \mu^{v}(\mathrm{~d} t, \mathrm{~d} x)\right) \mathrm{d} v \\
& =\int_{0}^{u} \mathrm{E}\left(\int_{0}^{\infty} \int_{\mathbb{R}} H(t, x) v^{v}(\mathrm{~d} t, \mathrm{~d} x)\right) \mathrm{d} v \\
& =\mathrm{E}\left(\int_{0}^{u}\left(\int_{0}^{\infty} \int_{\mathbb{R}} H(t, x) v^{v}(\mathrm{~d} t, \mathrm{~d} x)\right) \mathrm{d} v\right) \\
& =\mathrm{E}\left(\int_{0}^{\infty} \int_{\mathbb{R}} H(t, x) \bar{v}^{u}(\mathrm{~d} t, \mathrm{~d} x)\right),
\end{aligned}
$$

and the proof is complete.

Proposition A.4. Suppose that $M_{t}^{v}$ is a martingale for almost every $v \leq u$, and that $\mathrm{E}\left[\int_{0}^{u}\left|M_{t}^{v}\right| \mathrm{d} v\right]<\infty$ for all $t$. Then $\bar{M}_{t}^{u}=\int_{0}^{u} M_{t}^{v} \mathrm{~d} v$ is a martingale with $\Delta \bar{M}_{t}^{u}=\int_{0}^{u} \Delta M_{t}^{v} \mathrm{~d} v$.

Proof. A simple use of Fubini's theorem shows that, for $s<t$,

$$
\mathrm{E}\left[\bar{M}_{t}^{u} \mid \mathcal{F}_{s}\right]=\mathrm{E}\left[\int_{0}^{u} M_{t}^{v} \mathrm{~d} v \mid \mathcal{F}_{s}\right]=\int_{0}^{u} \mathrm{E}\left[M_{t}^{v} \mid \mathcal{F}_{s}\right] \mathrm{d} v=\int_{0}^{u} M_{s}^{v} \mathrm{~d} v=\bar{M}_{s}^{u} .
$$

In other words, $\bar{M}_{t}^{u}$ is a martingale.

Now recall that $M_{t-}=\mathrm{E}\left[M_{t} \mid \mathcal{F}_{t-}\right]$ for any martingale $M_{t}$. Thus,

$$
\bar{M}_{t-}^{u}=\mathrm{E}\left[\bar{M}_{t}^{u} \mid \mathcal{F}_{t-}\right]=\mathrm{E}\left[\int_{0}^{u} M_{t}^{v} \mathrm{~d} v \mid \mathcal{F}_{t-}\right]=\int_{0}^{u} \mathrm{E}\left[M_{t}^{v} \mid \mathcal{F}_{t-}\right] \mathrm{d} v=\int_{0}^{u} M_{t-}^{v} \mathrm{~d} v
$$

and the proof is complete.

The next proposition requires the following lemma, which is known to hold for continuous local martingales (see Revuz and Yor (1991, p. 120)).

Lemma A.1. Let $M$ and $N$ be two local martingales, denote by $\prec M, N \succ$ the total-variation process of $[M, N]$, and denote by $M^{\mathrm{c}}$ and $N^{\mathrm{c}}$ their continuous martingale parts. Then,

$$
\prec M, N \succ_{t} \leq 2 \sqrt{[M, M]_{t}[N, N]_{t}} .
$$

Proof. It follows from $[M, N]_{t}=\left\langle M^{\mathrm{c}}, N^{\mathrm{c}}\right\rangle_{t}+\sum_{s \leq t} \Delta M_{s} \Delta N_{s}$ that

$$
\begin{aligned}
\prec M, N \succ_{t} & \leq \prec M^{\mathrm{c}}, N^{\mathrm{c}} \succ_{t}+\sum_{s \leq t}\left|\Delta M_{s}\right|\left|\Delta N_{s}\right| \\
& \leq \sqrt{\left\langle M^{\mathrm{c}}, M^{\mathrm{c}}\right\rangle_{t}\left\langle N^{\mathrm{c}}, N^{\mathrm{c}}\right\rangle_{t}}+\sqrt{\sum_{s \leq t}\left(\Delta M_{S}\right)^{2} \sum_{s \leq t}\left(\Delta N_{s}\right)^{2}} \\
& \leq 2 \sqrt{[M, M]_{t}[N, N]_{t}} .
\end{aligned}
$$


Proposition A.5. Suppose that, for almost every $v \leq u, M_{t}^{v}$ is a (square-integrable) martingale such that $\mathrm{E}\left[\int_{0}^{u}\left\langle M^{v}, M^{v}\right\rangle_{\infty} \mathrm{d} v\right]<\infty$. Then $\bar{M}_{t}^{u}=\int_{0}^{u} M_{t}^{v} \mathrm{~d} v$ is a square-integrable martingale and

$$
\left\langle\bar{M}^{u}, \bar{M}^{u}\right\rangle_{t}=\int_{0}^{u} \int_{0}^{u}\left\langle M^{v}, M^{w}\right\rangle_{t} \mathrm{~d} v \mathrm{~d} w .
$$

Proof. First note that, for any $t$, both $\mathrm{E}\left[\left(\bar{M}_{t}^{u}\right)^{2}\right]$ and $\mathrm{E}\left[\int_{0}^{u}\left|M_{t}^{v}\right| \mathrm{d} v\right]^{2}$ are less than or equal to

$$
\begin{aligned}
\mathrm{E}\left[\left(\int_{0}^{u}\left|M_{t}^{v}\right| \mathrm{d} v\right)^{2}\right] & =\mathrm{E}\left[\int_{0}^{u} \int_{0}^{u}\left|M_{t}^{v} M_{t}^{w}\right| \mathrm{d} v \mathrm{~d} w\right]=\int_{0}^{u} \int_{0}^{u} \mathrm{E}\left[\left|M_{t}^{v} M_{t}^{w}\right|\right] \mathrm{d} v \mathrm{~d} w \\
& \leq \int_{0}^{u} \int_{0}^{u} \mathrm{E}\left[\left(M_{t}^{v}\right)^{2}\right]^{1 / 2} \mathrm{E}\left[\left(M_{t}^{w}\right)^{2}\right]^{1 / 2} \mathrm{~d} v \mathrm{~d} w=\left(\int_{0}^{u} \mathrm{E}\left[\left(M_{t}^{v}\right)^{2}\right]^{1 / 2} \mathrm{~d} v\right)^{2} \\
& \leq u \int_{0}^{u} \mathrm{E}\left[\left(M_{t}^{v}\right)^{2}\right] \mathrm{d} v=u \int_{0}^{u} \mathrm{E}\left[\left\langle M^{v}, M^{v}\right\rangle_{t}\right] \mathrm{d} v \\
& \leq u \mathrm{E}\left[\int_{0}^{u}\left\langle M^{v}, M^{v}\right\rangle_{\infty} \mathrm{d} v\right],
\end{aligned}
$$

which is finite. This and Proposition A.4 establish that $\bar{M}_{t}^{u}$ is a square-integrable martingale. Its sharp bracket is obtained as follows. First, Lemma A.1 shows that the total variation of the process $\left[M^{v}, M^{w}\right]_{t}$ is less than or equal to $2\left(\left[M^{v}, M^{v}\right]_{t}\left[M^{w}, M^{w}\right]_{t}\right)^{1 / 2}$. Since

$$
\mathrm{E}\left[\int_{0}^{u}\left[M^{v}, M^{v}\right]_{\infty} \mathrm{d} v\right]=\mathrm{E}\left[\int_{0}^{u}\left\langle M^{v}, M^{v}\right\rangle_{\infty} \mathrm{d} v\right]<\infty
$$

and

$$
\begin{aligned}
\int_{0}^{u} \int_{0}^{u} \sqrt{\left[M^{v}, M^{v}\right]_{t}\left[M^{w}, M^{w}\right]_{t}} \mathrm{~d} v \mathrm{~d} w & =\left(\int_{0}^{u} \sqrt{\left[M^{v}, M^{v}\right]_{t}} \mathrm{~d} v\right)^{2} \\
& \leq u \int_{0}^{u}\left[M^{v}, M^{v}\right]_{t} \mathrm{~d} v
\end{aligned}
$$

it follows from Proposition A.2 that $\int_{0}^{u} \int_{0}^{u}\left[M^{v}, M^{w}\right]_{t} \mathrm{~d} v \mathrm{~d} w$ is an integrable process of finite variation, the compensator of which is $\int_{0}^{u} \int_{0}^{u}\left\langle M^{v}, M^{w}\right\rangle \mathrm{d} v \mathrm{~d} w$. Finally, let $\tau$ be a finite stopping time. Then

$$
\begin{aligned}
\mathrm{E}\left[\left(\bar{M}_{\tau}^{u}\right)^{2}\right] & =\mathrm{E}\left[\int_{0}^{u} \int_{0}^{u} M_{\tau}^{v} M_{\tau}^{w} \mathrm{~d} v \mathrm{~d} w\right]=\int_{0}^{u} \int_{0}^{u} \mathrm{E}\left[M_{\tau}^{v} M_{\tau}^{w}\right] \mathrm{d} v \mathrm{~d} w \\
& =\int_{0}^{u} \int_{0}^{u} \mathrm{E}\left[\left[M^{v}, M^{w}\right]_{\tau}\right] \mathrm{d} v \mathrm{~d} w \\
& =\mathrm{E}\left[\int_{0}^{u} \int_{0}^{u}\left[M^{v}, M^{w}\right]_{\tau} \mathrm{d} v \mathrm{~d} w\right] \\
& =\mathrm{E}\left[\int_{0}^{u} \int_{0}^{u}\left\langle M^{v}, M^{w}\right\rangle_{\tau} \mathrm{d} v \mathrm{~d} w\right],
\end{aligned}
$$

which gives (A.1) and completes the proof.

Proposition A.6. Suppose that $M_{t}^{v}$ is continuous for almost every $v \leq u$, and that $\int_{0}^{u}\left\langle M^{v}, M^{v}\right\rangle_{t} \mathrm{~d} v<\infty$ for all $t$. Then $\bar{M}_{t}^{u}=\int_{0}^{u} M_{t}^{v} \mathrm{~d} v$ is a locally square-integrable martingale and (A.1) holds. 
Proof. Let $T_{n}=\inf \left\{t: \int_{0}^{u}\left\langle M^{v}, M^{v}\right\rangle_{t} \mathrm{~d} v>n\right\}$. Then, by monotone convergence,

$$
\int_{0}^{u}\left\langle M^{v}, M^{v}\right\rangle_{T_{n}} \mathrm{~d} v=\lim _{\varepsilon \downarrow 0} \int_{0}^{u}\left\langle M^{v}, M^{v}\right\rangle_{T_{n}-\varepsilon} \mathrm{d} v \leq n
$$

and the local martingales $M^{v}$, stopped at $T_{n}$, are square-integrable martingales (such that $\mathrm{E}\left[\left\langle M^{v}, M^{v}\right\rangle_{T_{n}}\right]<\infty$ almost everywhere) that satisfy the integrability condition of Proposition A.5. The result then follows from the application of Proposition A.5.

Proposition A.7. Suppose that, for almost every $v \leq u, M_{t}^{v}$ is a purely discontinuous local martingale such that the increasing process $\int_{0}^{u}\left[M^{v}, M^{v}\right]_{t} \mathrm{~d} v$ is locally integrable. Then $\bar{M}_{t-}^{u}=\int_{0}^{u} M_{t}^{v} \mathrm{~d} v$ is a locally square-integrable, purely discontinuous martingale such that $\Delta \bar{M}_{t}^{u}=\int_{0}^{u} \Delta M_{t}^{v} \mathrm{~d} v$, and (A.1) holds.

Proof. Let $T_{n}$ be such that

$$
\mathrm{E}\left[\int_{0}^{u}\left[M^{v}, M^{v}\right]_{T_{n}} \mathrm{~d} v\right]<\infty .
$$

Then, for almost every $v \leq u, M^{v}$ is a locally square-integrable martingale and

$$
\mathrm{E}\left[\int_{0}^{u}\left\langle M^{v}, M^{v}\right\rangle_{T_{n}} \mathrm{~d} v\right]<\infty .
$$

Therefore we can apply Proposition A.5 and Proposition A.4 to the local martingales $M^{v}$, stopped at $T_{n}$. We now establish that $\bar{M}^{u}$ is purely discontinuous. Let $L_{t}$ be a continuous, square-integrable (or even bounded) martingale and let $\tau$ be a finite stopping time. Then

$$
\mathrm{E}\left[\bar{M}_{\tau}^{u} L_{\tau}\right]=\mathrm{E}\left[\int_{0}^{u} M_{\tau}^{v} \mathrm{~d} v L_{\tau}\right]=\int_{0}^{u} \mathrm{E}\left[M_{\tau}^{v} L_{\tau}\right] \mathrm{d} v=0
$$

since $M^{v}$ is purely discontinuous and $L$ is continuous.

\section{Acknowledgements}

This research was done in part during the visit of Saul Jacka to the University of Melbourne, and was supported by the Australian Research Council and EPSRC.

\section{References}

BjöRK, T., KABAnOv, Y. AND RunggaldieR, W. (1997a). Bond market structure in the presence of marked point processes. Math. Finance 7, 211-239.

Björk, T., Di Masi, G., Kabanov, Y. and Runggaldier, W. (1997b). Toward a general theory of bond markets. Finance Stoch. 2, 141-174.

Cont, R. (1998). Modeling term structure dynamics: an infinite dimensional approach. To appear in Internat. J. Theoret. Appl. Finance.

Delbaen, F. And Schachermayer, W. (1994). A general version of the fundamental theorem of asset pricing. Math. Ann. 300, 463-520.

Hamza, K. AND Klebaner, F. C. (1995). A stochastic partial differential equation for term structure of interest rates. Res. Rep. SRR 016-94, University of Melbourne.

Harrison, J. M. AND Pliska, S. R. (1981). Martingales and stochastic integrals in the theory of continuous trading. Stoch. Process. Appl. 11, 215-260.

Heath, D. C., Jarrow, R. And Morton, A. (1990). Bond pricing and the term structure of interest rates: a discrete time approximation. J. Financial Quant. Anal. 25, 419-440. 
Heath, D. C., Jarrow, R. and Morton, A. (1992). Bond pricing and the term structure of interest rates: a new methodology for contingent claims valuation. Econometrica 60, 77-105.

JACOD, J. AND ShIRYAEv, A. N. (1987). Limit Theorems for Stochastic Processes. Springer, Berlin.

Kennedy, P. D. (1994). The term structure of interest rates as a Gaussian Markov field. Math. Finance 4, $247-258$.

LiptSER, R. Sh. AND ShIRYAev, A. N. (1989). Theory of Martingales. Kluwer, Dordrecht.

Musiela, M. And Sondermann, D. (1993). Different dynamical aspects of the term structure of interest rates and their implications. Res. Rep. B-260, SFB 303, Universität Bonn.

Revuz, D. ANd Yor, M. (1991). Continuous Martingales and Brownian Motion. Springer, Berlin.

ShiraKawa, H. (1991). Interest rate option pricing with Poisson-Gaussian forward rate curve process. Math. Finance 1, 77-94.

Shiryaev, A. N. (1999). Essentials of Stochastic Finance. World Scientific, River Edge, NJ.

Stricker, C. (1990). Arbitrage et lois de martingale. Ann. Inst. H. Poincaré Prob. Statist. 26, 451-460. 